\title{
Erratum to: Anticipatory Mobile Digital Health: Towards Personalized Proactive Therapies and Prevention Strategies
}

Veljko Pejovic, Abhinav Mehrotra and Mirco Musolesi

Erratum to:

Chapter "Anticipation Mobile Digital Health: Towards

Personalized Proactive Therapies and Prevention

Strategies" M. Nadin (ed.), Anticipation and Medicine, DOI 10.1007/978-3-319-45142-8_15

The original version of the chapter was inadvertently published without the updated corrections in the chapter "Anticipatory Mobile Digital Health: Towards Personalized Proactive Therapies and Prevention Strategies”. The erratum chapter has been updated with the changes.

The updated original online version this chapter can be found at http://dx.doi.org/10.1007/978-3319-45142-8_15 